\title{
Intraband carrier relaxation in quantum dots embedded in doped heterostructures
}

\author{
A. V. Baranov,* A. V. Fedorov, and I. D. Rukhlenko \\ S. I. Vavilov State Optical Institute, 12 Birzhevaya Liniya, 199034 St. Petersburg, Russia \\ Y. Masumoto \\ Institute of Physics, University of Tsukuba, Tsukuba 305-8571, Japan
}

(Received 7 April 2003; revised manuscript received 9 July 2003; published 21 November 2003)

\begin{abstract}
The effect of bulk plasmon-LO-phonon excitations inherent to doped areas of semiconductor heterostructures upon the electronic dynamics of quantum dots spaced apart from the areas is studied. An effective mechanism of intraband carrier relaxation in quantum dots is proposed for such heterostructures. The mechanism involves interaction between the quantum dot carriers and the electric potential induced by bulk plasmonLO-phonon modes of the doped areas. It is shown that the interaction opens two relaxation windows with the spectral positions and widths controlled, correspondingly, by the free-carrier concentrations and the bulk plasmon-LO-phonon mode dispersion of the doped areas. The relaxation rates related to the mechanism are calculated for quantum dots spaced apart by different distances $z_{0}$ from doped substrate (the area) with the different free-carrier concentrations $n_{0}$. The estimations carried out for InAs quantum dots and the GaAs substrate yield the relaxation rates of about $10^{8} \mathrm{~s}^{-1}$ for $n_{0}=10^{18} \mathrm{~cm}^{-3}$ and $z_{0}=100 \mathrm{~nm}$. The rates increase by two to three orders of magnitude with decreasing the distance down to $20 \mathrm{~nm}$. A manifestation of the mechanism is shown for a system of self-assembled InAs/GaAs quantum dots separated by a distance of $100 \mathrm{~nm}$ from an $n$-doped GaAs substrate by means of the photoluminescence spectroscopy. From the experiment it has been found that $L^{-}$and $L^{+}$plasmon-LO-phonon modes of the GaAs substrate are involved in the intraband carrier relaxation in the quantum dots. The data are in good agreement with the theoretical predictions.
\end{abstract}

DOI: 10.1103/PhysRevB.68.205318 PACS number(s): 78.66.Fd, 63.22.+m, 71.35.Cc, 78.55.Cr

\section{INTRODUCTION}

Rapid development of nanoengineering allows one to design quantum dot based nanoelectronic devices with a lot of structural components included doped semiconductor layers, connection elements, and substrates. The single electron transistors, ${ }^{1}$ quantum bits, ${ }^{2}$ memory storage cells, ${ }^{3}$ and quantum dot lasers ${ }^{4}$ are the typical examples of such devices. Since the quantum dots (QD's) are their major operating units, an intimate knowledge about energy and phase relaxation of the QD electronic subsystem is quite necessary to manufacture the high-performance devices. Up to now the main research efforts in this field have been directed to a study of relaxation processes caused by interactions with different elementary excitations localized inside the quantum dot or at its interface. For example, the effects of confined and interface optical phonons ${ }^{5-10}$ and plasmons ${ }^{11-13}$ on the QD electronic dynamics have been investigated. Several works have been devoted to study a multiphonon mediated relaxation involving longitudinal optical (LO) and acoustic phonons. ${ }^{8,10,14,15}$ The defect-assisted multiphonon emission mechanism ${ }^{16-18}$ has been proposed for an explanation of a fast carrier relaxation in quantum dots. Moreover, the Augerlike process ${ }^{19,20}$ has been considered as another effective mechanism of intraband carrier relaxation.

In spite of the fact that the real QD based devices are the complicated heterostructures composed of many structure components (e.g., the host matrix, the quantum wells and wires, the capping, buffer, and wetting layers, etc.) there are relatively few studies of the QD electronic dynamics affected by interactions with the environment elementary excitations. Besides the interaction between QD electronic subsystem and barrier/matrix optical and acoustical phonons, ${ }^{7,8,21-23}$ the influence of only nearest surroundings on the QD dynamics has been studied so far. For example, homogeneous broadening of optical transitions in self-assembled quantum dots caused by the elastic Coulomb collisions between carriers in wetting layer and in the dots ${ }^{24}$ has been considered. The QD electronic dephasing caused by the charge fluctuations in an impurity state due to its recharging through the free-electron reservoir ${ }^{2}$ have been investigated. It may be expected that not only free charges of environment but also plasmons and plasmon phonons which reside in doped heterostructure components will interact with the QD electronic subsystem. Evidently at close contact of the dots with the doped components the QD carriers will strongly interact with environment excitations accompanied by the electric fields. But in many cases such components, e.g., doped substrates, are remote from the quantum dots by several ten nanometers and strength of the interaction is a problem of question.

Simple electrostatic considerations show that the electric field induced by longitudinal bulk waves regardless of their nature (LO phonons, plasmons or plasmon-LO-phonon modes) cannot penetrate to materials with another dielectric permittivity. ${ }^{25,26}$ However, it has been shown by the example of the LO phonons ${ }^{27,28}$ that account of the phonon dispersion results in a rise of exponential tails of electric field in another material. The same situation is expected to take place in the case of the plasmons or plasmon-LO-phonon modes. Their dispersion plays a drastic role for understanding the physics of intraband carrier relaxation in quantum dots remote from doped material. It is due to the dispersion that electric fields induced by the longitudinal bulk waves can penetrate through interface and affect the QD electronic subsystem opening new relaxation channels.

In this work we develop a theoretical model of the QD 


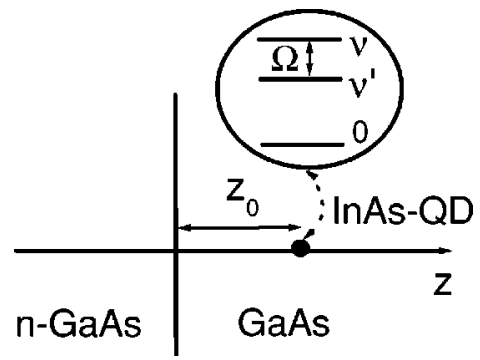

FIG. 1. A heterostructure considered in the model. Electron structure of quantum dot is shown schematically. $\Omega$ is the QD intraband transition frequency, $z_{0}$ is the distance between the doped semiconductor and dot.

carrier intraband relaxation via interaction between the carriers and the electric potential induced by the bulk plasmonLO-phonon (PLP) modes of doped heterostructure components. We report experimental observation of the mechanism in system of InAs/GaAs self-assembled quantum dots (SAQD's). We show that the mechanism can be used for manipulation of electronic dynamics in QD based devices with $n(p)$-doped substrates.

\section{THEORETICAL MODEL}

In order to understand a physical reason of coupling between the QD carriers and the bulk plasmon-LO-phonon modes of doped structural component we consider a simple model of heterostructure composed of two half-spaces filled by doped and undoped semiconductor with a plane interface (Fig. 1). The quantum dot is located at a distance $z_{0}$ from the doped material. To find the electric potential induced by the bulk PLP modes at the dot position we use an approach close to that which has been employed earlier ${ }^{29}$ for description of the plasmon-photon modes in a single heterostructure. Such type of approach is based on the motion equations for all quasiparticles and fields under consideration. In our case the Bloch hydrodynamic equations describing the motion of an electron gas coupled with the electric field ${ }^{29-31}$ is supplemented by the equation for dispersionless optical phonons: ${ }^{26}$

$$
\begin{gathered}
\frac{\partial \psi}{\partial t}=\frac{1}{2}(\nabla \psi)^{2}-\frac{e}{m} \varphi+\frac{1}{m} \int_{0}^{n} \frac{d p\left(n^{\prime}\right)}{n^{\prime}}, \\
\frac{\partial n}{\partial t}=\nabla \cdot(n \nabla \psi), \\
\Delta \varphi=\frac{4 \pi e}{\varepsilon_{\infty}}\left(n-N_{0}\right)+\frac{4 \pi \alpha}{\varepsilon_{\infty}} \nabla \cdot \mathbf{u}, \\
\frac{\partial^{2} \mathbf{u}}{\partial t^{2}}=-\omega_{T}^{2} \mathbf{u}-\frac{\alpha}{\rho} \nabla \varphi,
\end{gathered}
$$

where $\psi$ is the potential of the hydrodynamic velocity $\mathbf{v}=-\nabla \psi$ of the electron gas, $n$ is the density of free carriers with effective mass $m, \varphi$ is the self-consisted electric potential, $p(n)$ is the pressure-density relation of the carrier gas, $\mathbf{u}$ is the mechanical displacement field corresponding of optical phonons, $\alpha=\omega_{T}\left[\left(\varepsilon_{0}-\varepsilon_{\infty}\right) \rho / 4 \pi\right]^{1 / 2}, \varepsilon_{0}$ and $\varepsilon_{\infty}$ are the low- and high-frequency dielectric constants, $\rho$ is the reducedmass density of a unit cell, $\omega_{T}$ is the limiting frequency of transversal optical phonons, and $N_{0}$ is the dopant concentration. In contrast to the polaritonic problem we take into account only the longitudinal electric field $\mathbf{E}=-\nabla \varphi$ and include to the Poisson equation [see Eq. (3)] an additional term that describes contribution of the phonon induced lattice polarization to the charge density. According to Eqs. (1)-(4) the fluctuations of electron gas density are coupled with the longitudinal optical phonons via the electric potential $\varphi$. It is easy to see that the phenomenological Lagrangian corresponding to Eqs. (1)-(4) has the following form:

$$
\begin{aligned}
L= & \int d^{3} r\left[\frac{1}{2} \rho \dot{\mathbf{u}}^{2}-\frac{1}{2} \rho \omega_{T}^{2} \mathbf{u}^{2}-\alpha \nabla \varphi \cdot \mathbf{u}+\frac{\varepsilon_{\infty}}{8 \pi}(\nabla \varphi)^{2}+m n \dot{\psi}\right. \\
& \left.-\frac{1}{2} m n(\nabla \psi)^{2}+e \varphi\left(n-N_{0}\right)-n \int_{0}^{n} d n^{\prime} \frac{p\left(n^{\prime}\right)}{n^{\prime 2}}\right] .
\end{aligned}
$$

The Lagrangian (5) allows us to define the appropriate Hamiltonian and by means of its diagonalization to introduce the eigen-PLP modes as well as an interaction between the electron subsystem of QD's and the PLP modes. Before proceeding to this program we simplify our problem using the usual linearization procedure. ${ }^{29-31}$ As result, instead of Eqs. (1)-(4), a set of linear equations,

$$
\begin{gathered}
\frac{\partial \psi}{\partial t}=-\frac{e}{m} \varphi+\frac{\beta^{2}}{n_{0}} n, \\
\frac{\partial n}{\partial t}=\nabla \cdot\left(n_{0} \nabla \psi\right), \\
\Delta \varphi=\frac{4 \pi e}{\varepsilon_{\infty}} n+\frac{4 \pi \alpha}{\varepsilon_{\infty}} \nabla \cdot \mathbf{u}, \\
\frac{\partial^{2} \mathbf{u}}{\partial t^{2}}=-\omega_{T}^{2} \mathbf{u}-\frac{\alpha}{\rho} \nabla \varphi,
\end{gathered}
$$

is obtained and the corresponding Hamiltonian is given by

$$
\begin{aligned}
H= & \int d^{3} r\left[\frac{1}{2} \rho \dot{\mathbf{u}}^{2}+\frac{1}{2} \rho \omega_{T}^{2} \mathbf{u}^{2}+\alpha \nabla \varphi \cdot \mathbf{u}-\frac{\varepsilon_{\infty}}{8 \pi}(\nabla \varphi)^{2}\right. \\
& \left.+\frac{1}{2} m n_{0}(\nabla \psi)^{2}-e \varphi n+\frac{m \beta^{2}}{2 n_{0}} n^{2}\right],
\end{aligned}
$$

where $n_{0}=N_{0}$ is the uniform electron gas density in the undisturbed state and $\beta=\left[\hbar^{2}\left(3 \pi^{2} n_{0}\right)^{2 / 3} / 3 m^{2}\right]^{1 / 2}$ is the speed of propagation of hydrodynamic disturbance in the electron gas. ${ }^{29}$ In order to obtain the eigen-PLP modes we solved Eqs. (6)-(9) for the doped $(d)$ and undoped $(u)$ parts of heterostructure provided an equality to zero of the normal component of hydrodynamic velocity of electron gas $d \psi_{d} /\left.d z\right|_{z=-0}=0$ as well as a continuity of the selfconsisting electric potential $\left.\varphi_{d}\right|_{z=-0}=\left.\varphi_{u}\right|_{z=+0}$ and the normal component of electric displacement $\varepsilon_{d}(\omega) d \varphi_{d} /\left.d z\right|_{z=-0}=\varepsilon_{u}(\omega) d \varphi_{u} /\left.d z\right|_{z=+0}$, where $\varepsilon_{i}(\omega)$ $=\varepsilon_{i \infty}\left(\omega^{2}-\omega_{i L}^{2}\right) /\left(\omega^{2}-\omega_{i T}^{2}\right)$ for $i=d$ or $u$, and $\omega_{i L}$ are the limiting frequencies of longitudinal optical phonons. Analy- 


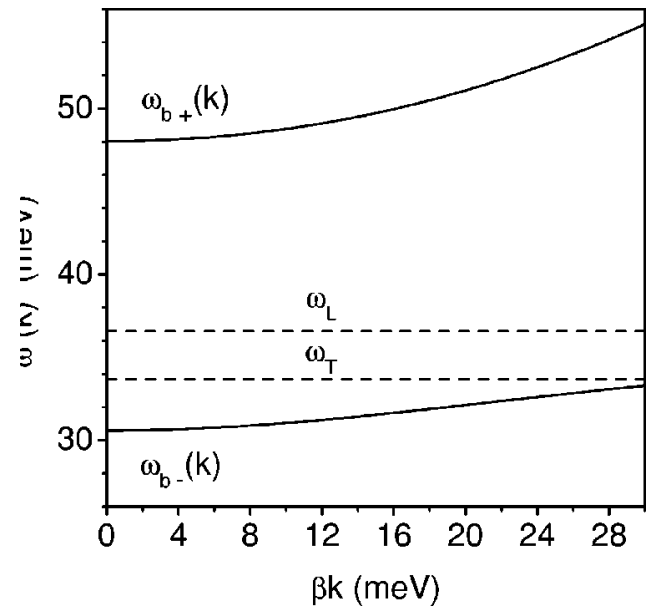

FIG. 2. The dispersion brunches for the bulk PLP modes in $n$-doped GaAs substrate with the concentration of free electrons equal $n_{0}=10^{18} \mathrm{~cm}^{-3}$.

sis has shown that the bulk plasmon-LO-phonon modes occur if their wave vector $\mathbf{k}$ and frequency $\omega$ satisfy the inequality

$$
\beta^{2} k_{z}^{2}=\omega^{2}-\omega_{p}^{2} \frac{\varepsilon_{\infty}}{\varepsilon(\omega)}-\beta^{2} \mathbf{q}^{2}>0,
$$

where $k_{z}$ is the normal component of $\mathbf{k}, \mathbf{q}$ is the projection of $\mathbf{k}$ onto the interface plane, $\omega_{p}=\sqrt{4 \pi n_{0} e^{2} / \varepsilon_{\infty} m}$ is the plasma frequency, and all material parameters correspond to the doped semiconductor. Then we obtain that the bulk plasmonphonon modes have two branches of a dispersion relation (Fig. 2) with frequencies

$$
\begin{aligned}
\omega_{b \pm}^{2}(k)= & \frac{1}{2}\left\{\omega_{L}^{2}+\omega_{p}^{2}+\beta^{2} k^{2} \pm\left[\left(\omega_{L}^{2}+\omega_{p}^{2}+\beta^{2} k^{2}\right)^{2}\right.\right. \\
& \left.\left.-4 \omega_{p}^{2} \omega_{T}^{2}-4 \omega_{L}^{2} \beta^{2} k^{2}\right]^{1 / 2}\right\} .
\end{aligned}
$$

Obviously the upper branch of the plasmon-phonon modes is restricted from below by the condition $\omega_{b+}(k)$ $\geqslant \omega_{b+}(0)$ while the lower branch is restricted by the double inequality $\omega_{T}>\omega_{b-}(k) \geqslant \omega_{b-}(0)$. Using the solution of Eqs. (6)-(9) and the Hamiltonian (10) we found a coupling energy $V(\mathbf{r})=-e \varphi(\mathbf{r})$ between the QD electronic subsystem and the self-consisted electric potential $\varphi(\mathbf{r})$ induced by the PLP modes as

$$
V(\mathbf{r})=\sum_{\mathbf{k}}\left[V_{\mathbf{k}}(z) e^{i \mathbf{q} \cdot \mathbf{x}} b_{\mathbf{k}}+V_{\mathbf{k}}^{*}(z) e^{-i \mathbf{q} \cdot \mathbf{x}} b_{\mathbf{k}}^{+}\right],
$$

where $b_{\mathbf{k}}\left(b_{\mathbf{k}}^{+}\right)$is the annihilation (creation) operator of the plasmon-phonon mode and $\mathbf{x}$ is the coordinate in the interface plane. As for a function $V_{\mathbf{k}}(z)$, it has the simplest form when both parts of the heterostructure are filled by the same materials like the structure shown in Fig. 1. Then

$$
V_{\mathbf{k}}(z)=V_{k} \cdot \begin{cases}\zeta_{\mathbf{k}} e^{i k_{z} z}+e^{-i k_{z} z}-\chi_{\mathbf{k}} \omega^{2} e^{q z}, & z \leqslant 0, \\ \chi_{\mathbf{k}} \beta^{2} k^{2} e^{-q z}, & z>0,\end{cases}
$$

where

$$
\begin{gathered}
\zeta_{\mathbf{k}}=\frac{i k_{z} \delta(\omega)+q \gamma(\omega)}{i k_{z} \delta(\omega)-q \gamma(\omega)}, \quad \chi_{\mathbf{k}}=\frac{i k_{z}}{i k_{z} \delta(\omega)-q \gamma(\omega)}, \\
\gamma(\omega)=\frac{\omega_{p}^{2}}{2} \frac{\omega^{2}-\omega_{T}^{2}}{\omega^{2}-\omega_{L}^{2}}, \quad \delta(\omega)=\beta^{2} k^{2}+\gamma(\omega)
\end{gathered}
$$

$V_{k}=\left[2 \hbar m \gamma^{2}(\omega) / L^{3} k^{2} n_{0} \omega \sigma(\omega)\right]^{1 / 2}, L^{3}$ is the normalized volume, $\quad \sigma(\omega)=1+\omega_{p}^{2}\left(\omega_{L}^{2}-\omega_{T}^{2}\right) /\left(\omega^{2}-\omega_{L}^{2}\right)^{2}, \quad \omega$ equals $\omega_{b+}(k)$ or $\omega_{b-}(k)$ depending on the considered PLP branch. The lower string of Eq. (14) shows that the self-consistent electric potential penetrates to the undoped part of the heterostructure and is responsible for the interaction between the QD electron subsystem and the plasmon-phonon modes. Moreover, as we mentioned above, the coupling vanishes for dispersionless PLP modes $(\beta=0)$.

Now, using the Fermi golden rule, we can calculate the intraband relaxation rates of QD electrons due to interaction with the doped semiconductor via electric potential induced by the plasmon-phonon modes. Supposing that temperatures are relatively small, $\omega_{b \pm}(k) / k_{B} T \gg 1$, we can restrict our consideration to only relaxation processes with emission of the PLP modes. Then, the rate of the intraband electron transitions depending on the intraband QD level spacing $\Omega$ $=\left(E_{\nu}-E_{\nu^{\prime}}\right) / \hbar$, where $E_{\nu\left(\nu^{\prime}\right)}$ is the energy of initial (final) QD state with the quantum numbers $\nu\left(\nu^{\prime}\right)$ (see Fig. 1), is given by

$$
W_{b \pm}^{\left(\nu^{\prime}, \nu\right)}=\frac{m \beta^{2} q_{\Omega}^{3}}{\pi \hbar n_{0}} \int_{0}^{1} \frac{d \tau e^{-2 q_{\Omega} z_{0} \tau} \tau \sqrt{1-\tau^{2}} f_{\nu^{\prime}, \nu}(\tau)}{1+a(\Omega)\left(1-\tau^{2}\right)},
$$

where

$$
q_{\Omega}=\frac{1}{\beta} \sqrt{\left[\Omega^{2}-\omega_{b+}^{2}(0)\right]\left[\Omega^{2}-\omega_{b-}^{2}(0)\right] /\left(\Omega^{2}-\omega_{L}^{2}\right)},
$$

$a(\Omega)=\left[\Omega \beta q_{\Omega} / \gamma(\Omega)\right]^{2}$, and the function $f_{\nu^{\prime}, \nu}(\tau)$ containing all information about QD parameters is

$$
f_{\nu^{\prime}, \nu}(\tau)=A v_{\eta^{\prime}, \eta}\left|\left\langle\nu^{\prime}, \eta^{\prime}\left|e^{-q_{\Omega} \tau(z-i|\mathbf{x}| \cos \psi)}\right| \nu, \eta\right\rangle\right|^{2},
$$

where $\psi$ is the angle between $\mathbf{q}$ and $\mathbf{x}$ vectors. The operation $A v_{\eta^{\prime}, \eta}$ in Eq. (19) implies the averaging over degenerate initial QD states $|\nu, \eta\rangle$ and summation over degenerate final QD states $\left|\nu^{\prime}, \eta^{\prime}\right\rangle$, where $\eta$ and $\eta^{\prime}$ are the quantum numbers related to the degenerated QD states. For example, for a spherical QD of the radius $R_{0}$ in the strong confinement regime, ${ }^{32}$ i.e., when $R_{e x}>R_{0}$, where $R_{e x}$ is the bulk exciton Bohr radius for the QD material,

$$
f_{n^{\prime} l^{\prime}, n l}(\tau)=2 \sum_{p=0}^{\infty}\left(2 R_{0} q_{\Omega} \tau\right)^{2 p} \frac{\left(J_{n^{\prime} l^{\prime}, n l}^{(p)} C_{l 0, p 0}^{l^{\prime} 0}\right)^{2}}{(2 p+1) !}
$$

where $n l\left(n^{\prime} l^{\prime}\right)$ are the principle quantum number and angular momentum of initial (final) QD electronic states, $C_{l m, p t}^{l^{\prime} m^{\prime}}$ are the Clebsch-Gordon coefficients ${ }^{33}$ governing the transition selection rules. The quantities $J_{n^{\prime} l^{\prime}, n l}^{(p)}$ for a quantum dot with an infinitely high potential barrier $\left(E_{0} \rightarrow \infty\right)$ equal 


$$
J_{n^{\prime} l^{\prime}, n l}^{(p)}=2 \int_{0}^{1} d x x^{2+p} \frac{j_{l^{\prime}}\left(\xi_{n^{\prime} l^{\prime}} x\right) j_{l}\left(\xi_{n l} x\right)}{j_{l^{\prime}+1}\left(\xi_{n^{\prime} l^{\prime}}\right) j_{l+1}\left(\xi_{n l}\right)},
$$

where $\xi_{n l}$ is the $n$th root of the spherical Bessel function of $l$ th order $\left[j_{l}\left(\xi_{n l}\right)=0\right]$. If the potential barrier $E_{0}$ is finite then

$$
\begin{gathered}
J_{n^{\prime} l^{\prime}, n l}^{(p)}=2 A_{n^{\prime} l^{\prime}} A_{n l}\left(B_{n^{\prime} l^{\prime}}^{n l} \int_{0}^{1} d x x^{2+p} j_{l^{\prime}}\left(\eta_{n^{\prime} l^{\prime}} x\right) j_{l}\left(\eta_{n l} x\right)\right. \\
\left.+C_{n^{\prime} l^{\prime}}^{n l} \int_{1}^{\infty} d x x^{2+p} k_{l^{\prime}}\left(\zeta_{n^{\prime} l^{\prime}} x\right) k_{l}\left(\zeta_{n l} x\right)\right) \\
A_{n l}=\left[j_{l}^{2}\left(\eta_{n l}\right) k_{l-1}\left(\zeta_{n l}\right) k_{l+1}\left(\zeta_{n l}\right)\right. \\
\left.\quad-k_{l}^{2}\left(\zeta_{n l}\right) j_{l-1}\left(\eta_{n l}\right) j_{l+1}\left(\eta_{n l}\right)\right]^{-1 / 2} \\
B_{n^{\prime} l^{\prime}}^{n l}=k_{l^{\prime}}\left(\zeta_{n^{\prime} l^{\prime}}\right) k_{l}\left(\zeta_{n l}\right), C_{n^{\prime} l^{\prime}}^{n l}=j_{l^{\prime}}\left(\eta_{n^{\prime} l^{\prime}}\right) j_{l}\left(\eta_{n l}\right)
\end{gathered}
$$

where $k_{l}$ is the modified spherical Bessel function, $\eta_{n l}$ $=R_{0}\left(2 m_{1} E_{n l} / \hbar^{2}\right)^{1 / 2}, \quad \zeta_{n l}=R_{0}\left[2 m_{2}\left(E_{0}-E_{n l}\right) / \hbar^{2}\right]^{1 / 2}$, $m_{1}\left(m_{2}\right)$ is the electronic effective mass in the dot (surrounding media), the electronic energy $E_{n l}$ is determined by the secular equation ${ }^{34}$

$$
m_{2} \eta_{n l} k_{l}\left(\zeta_{n l}\right) j_{l}^{\prime}\left(\eta_{n l}\right)=m_{1} \zeta_{n l} k_{l}^{\prime}\left(\zeta_{n l}\right) j_{l}\left(\eta_{n l}\right)
$$

Equation (17) describes two relaxation windows resulting from the intraband transitions with emission of the PLP modes of upper dispersion branch $\omega_{b+}(k)$ if $\Omega \geqslant \omega_{b+}(0)$ and those with emission of the PLP modes of lower dispersion branch $\omega_{b-}(k)$ if $\omega_{T}>\Omega \geqslant \omega_{b-}(0)$ (see Fig. 2). It is clearly seen that spectral positions of the windows depend on free-carrier concentration in doped structural component, and spectral widths of the windows and values of the relaxation rates are determined by the dispersion of the plasmonphonon modes.

\section{EXPERIMENTAL RESULTS AND DISCUSSION}

The discussed coupling of QD electronic subsystem with PLP modes of $n$-doped substrate and the intraband carrier relaxation mediated by these modes have been experimentally observed at $2 \mathrm{~K}$ in two systems of InAs/GaAs SAQD's with different parameters of electronic states. Two samples (A and B) were fabricated by MBE on a (001) surface of a Si-doped $\left(n_{0} \approx 10^{18} \mathrm{~cm}^{-3}\right)$ GaAs substrate. In both cases a 100-nm GaAs buffer layer was grown. Then InAs of 1.8 monolayers (ML's) was deposited at $500{ }^{\circ} \mathrm{C}\left(480{ }^{\circ} \mathrm{C}\right)$ giving rise to lens-shaped QD's with diameter of $\approx 22(18) \mathrm{nm}$, height of $\approx 10(8) \mathrm{nm}$, and an areal density of $\approx 1.2(2)$ $\times 10^{10} \mathrm{~cm}^{-2}$ for sample A (B). The thickness of a GaAs cap layer was $150 \mathrm{~nm}$. The electronic energy structure of both quantum dot systems was found from their photoluminescence (PL) spectra in standard state-filling experiments ${ }^{35}$ with excitation by a 514.5-nm line of $\mathrm{Ar}^{+}$laser. It was typical for lens-shaped InAs SAQD's (Ref. 36) with quasiequidistant spectra of interband inhomogeneously broadened $(\approx 70 \mathrm{meV})$ transitions and peak energies of the lowest $(0-0)$ transitions of $\approx 1228 \mathrm{meV}(\mathrm{A})$ and $\approx 1321 \mathrm{meV}(\mathrm{B})$. Insets in lower and upper panels in Fig. 3 show the PL spectra of

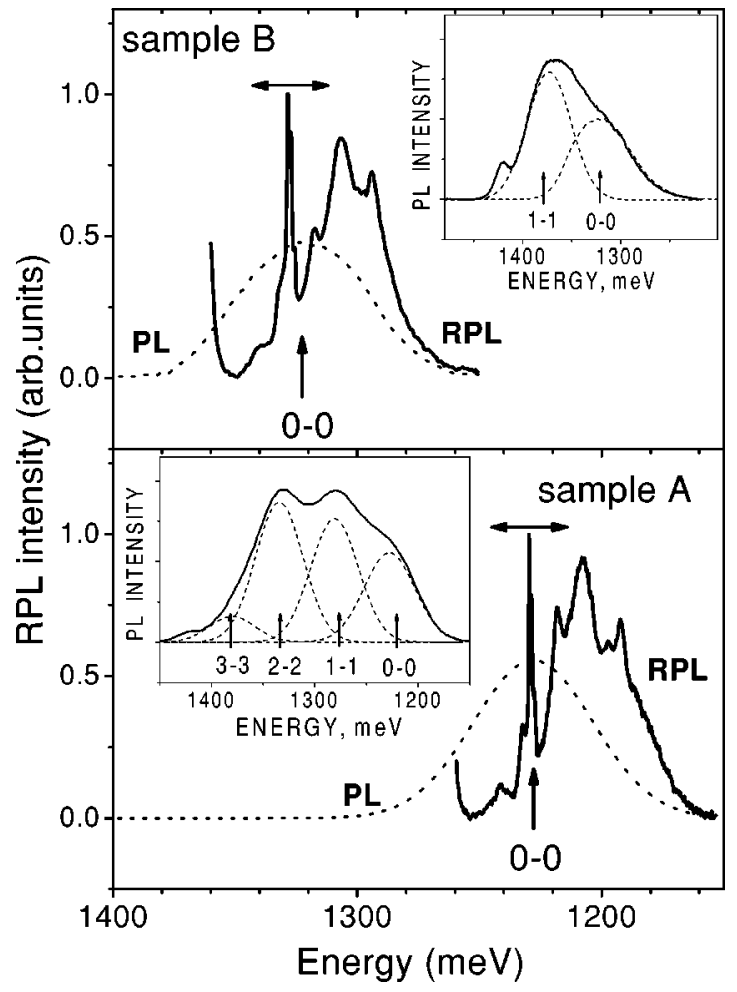

FIG. 3. The resonant photoluminescence (RPL) spectrum of InAs SAQD's in samples A (lower panel) and B (upper panel) excited in resonance with the 1-1 transition of quantum dots (1262 and $1361 \mathrm{meV}$, respectively). The position of the $0-0$ photoluminescence (PL) band of the dots (dotted curve) is shown for comparison in both panels. The insets present the PL spectra of the samples measured in the state-filling experiments (see the text). A Gaussian line-shape fit is shown. The positions of the SAQD interband transitions (0-0, 1-1, etc.) are shown by arrows. The horizontal arrow bar shows the portion of spectrum which is under detailed analysis.

the samples at high excitation power and fitting of the PL spectra by a sum of Gaussians. The peak maximums correspond to the sequence of the QD interband transitions (0-0, $1-1$, etc.). Energy gaps between peaks of the lowest and second transitions were about $53 \mathrm{meV}$ (A) and $55 \mathrm{meV}$ (B). Taking into account the inhomogeneous broadening of the transitions we concluded that the energies of the QD intraband level spacing ${ }^{36}$ match those of the PLP modes expected for studied quantum dot heterostructures.

The secondary emission spectra of the samples were measured in the $x(y, y) z$ geometry, where $z$ is the growth direction of the InAs layer, by the use of a cw wavelength-tunable Ti:sapphire laser and a double monochromator equipped by a cooled InAs photomultiplier. The measurements were done at low pump power $\left(<0.2 \mathrm{~W} / \mathrm{cm}^{2}\right)$ when PL is only caused by recombination of the electrons and holes in their ground states. It was found that in accordance with earlier data ${ }^{8,15,37}$ the PL spectra of the studied QD samples excited in resonance with high-energy QD transitions (hereinafter referred to as RPL spectra) are mainly formed by lines resulting from one- and multiphonon intraband carrier relaxation in the quantum dots. As an example, the RPL spectra of samples A and $\mathrm{B}$ excited in resonance with 1-1 transitions (the photon energies of 1262 and $1361 \mathrm{meV}$, respectively) are shown in 


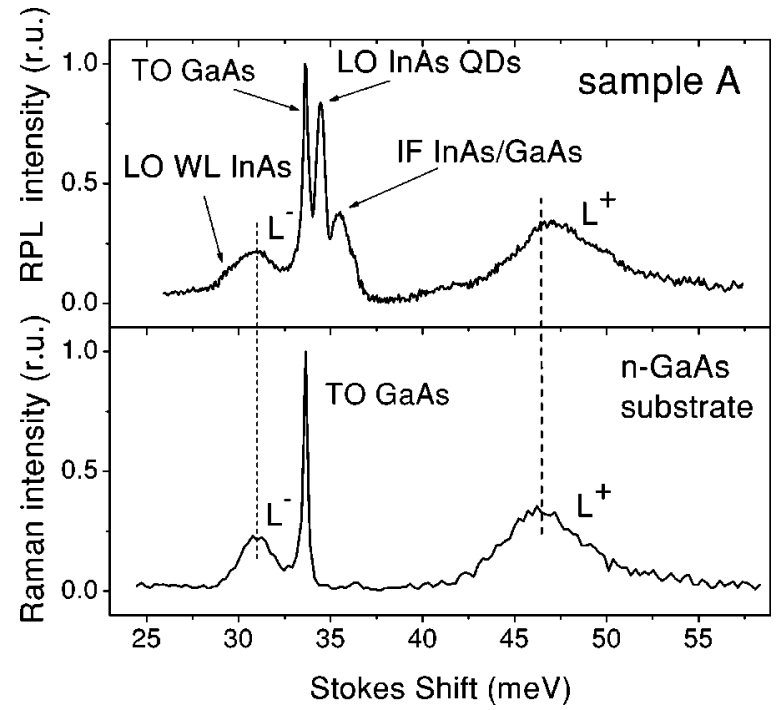

FIG. 4. Upper panel: Enlarged part of the RPL spectrum of sample A marked in Fig. 3 (lower panel) by the arrow bar plotted vs the Stokes shift. The background caused by the multiphonon lines was subtracted. Lower panel: The Raman spectrum of the $n$-doped GaAs substrate obtained from area of sample A with removed quantum dots. The spectrum has measured at the same excitation/ detection conditions as the RPL one. The assignment of the lines in the RPL and Raman spectra is shown (see the text).

Fig. 3. To make an assignment of the RPL spectrum lines, the spectra were measured at different incident photon energies $E_{e x}$ covering the interband transition energies of QD's and were analyzed as a function of the Stokes shift, $E_{e x}$ $-E_{R P L}$, where $E_{R P L}$ is the energy of photons detected in RPL experiments. The RPL spectra were fitted by a sum of Gaussians, that yields parameters of the overlapping lines. ${ }^{8,15,37}$ It has been found that the Stokes shifts of the lines are invariable. This is a sign of phonon-assistant resonant photoluminescence. The lines of InAs wetting layer (WL), intrinsic LO phonons of InAs QD's, InAs/GaAs phonon interface mode (IF) with the Stokes shifts of 29.5, 34.3, and $35.5 \mathrm{meV}$, respectively, as well as their overtones and sum tones have been easily distinguished under analysis of the spectra. Assignment of the lines was carried out according to Ref. 7 where resonant photoluminescence spectra of the analogous InAs SAQD sample were studied. Further, we have analyzed in more detail the obtained RPL spectra in region of the Stokes shifts of $25-60 \mathrm{meV}$, as is shown in Fig. 3 by the arrow bars. This region of the spectrum after subtraction of background caused by the multiphonon lines is plotted vs Stokes shift in Fig. 4 (upper panel) for sample A. It is seen that except for the above mentioned phonon lines, the lines belonging to the zone-center bulk TO phonons and to the PLP modes, $L^{-}$, and $L^{+}$(corresponding energies $E_{L^{-}}$ and $E_{L^{+}}$are 31.1 and $46.7 \mathrm{meV}$, respectively) of $n$-doped GaAs (Ref. 38) appear to be in the spectra. The assignment of the $L^{-}$and $L^{+}$lines is based on comparison of the resonant photoluminescence spectra with Raman spectra of $n$-doped GaAs substrate. The Raman spectra were obtained from area of the sample with removed quantum dots at the same excitation/detection conditions as the RPL spectra. An example of the Raman spectrum is shown in Fig. 4 (low

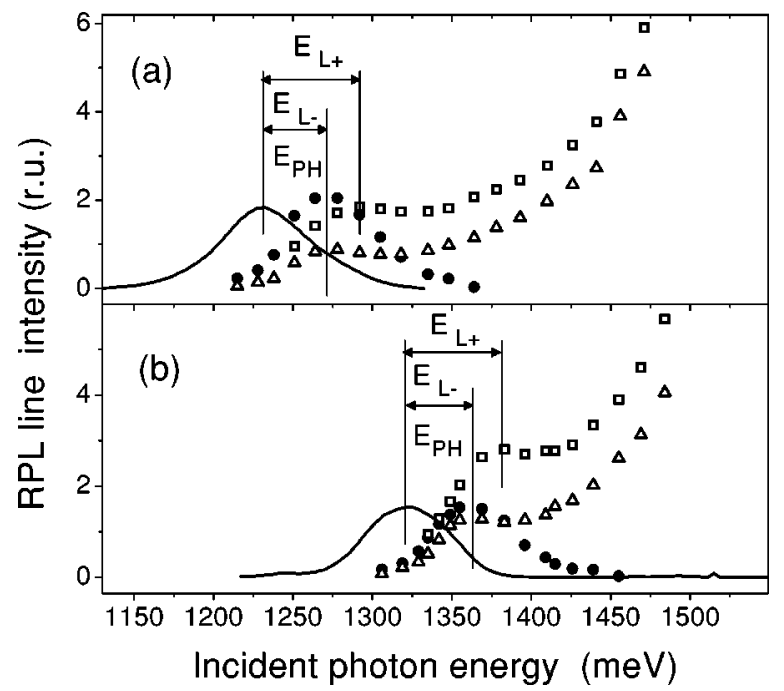

FIG. 5. Excitation profiles of the $L^{+}$(open squares) and $L^{-}$ (open triangles) lines observed in the RPL spectra of samples A and B [(a) and (b), respectively]. The filled circles show the excitation profile of the intrinsic LO phonon line of InAs QD's. Solid curves show the positions of the QD 0-0 PL bands. $E_{P H}, E_{L^{-}}$, and $E_{L^{+}}$are the energies of the LO phonons of InAs QD's, $L^{-}$and $L^{+}$PLP modes, respectively.

panel). The resonant photoluminescence spectra of sample B exhibited the same features. At first glance, the spectrum presented in the upper panel of Fig. 4 is a simple superposition of the phonon-assisted RPL spectrum of the quantum dots and Raman spectrum of the substrate. However, it was found that plasmon-LO-phonon lines in spectra of samples with QD's show unusual behaviors which indicate coupling between the substrate plasmon-phonon modes and electronic subsystem of the quantum dots.

We have plotted the integral intensities of the $L^{-}$and $L^{+}$ lines of the samples with quantum dots as a function of the incident photon energy, so-called excitation profiles of the lines commonly used in resonant Raman spectroscopy. The excitation profiles of these lines in the RPL spectra of QD's are presented in Fig. 5 for samples A (a) and B (b) together with excitation profile of the intrinsic LO-phonon line of QD's. The positions of the 0-0 PL bands are shown in the same figure for comparison (solid curves). They indicate the energy spectra of inhomogeneously broadened lowest interband transitions of the QD ensembles. As can be seen, the excitation profile of the LO-phonon line of InAs QD's demonstrates single prominent peak blueshifted by the LOphonon energy $\left(E_{P H}\right)$ with respect to the $0-0$ band. The excitation profiles of other phonon lines and their overtones demonstrated analogous shifts by the corresponding phonon or overtone energies. That is a signature of the fact that the phonon lines come from annihilation of the electron-hole pairs in the lowest energy state populated by the phononmediated intraband carrier relaxation from the directly photoexcited state. This process is described in detail in Refs. 12 and 35. In this case the intensity of the phonon lines excited by radiation with photon energy tunable in region of the higher-energy transitions of QD's follows the 0-0 PL band contour. It is just our case that can be seen if the excitation 
profile in Fig. 5 will be shifted by the LO phonon energy to the red side. Evidently the analysis of the RPL line excitation profiles is equivalent to that of photoluminescence excitation spectra.

Let us discuss the excitation profiles of the $L^{-}$and $L^{+}$ lines. As is well known, ${ }^{39}$ in $n$-doped bulk GaAs the intensities of the $L^{-}$, and $L^{+}$Raman lines have to increase monotonically with excitation energy approaching the GaAs band gap (pre-resonant Raman). However, except for this, we found that the excitation profiles of the $L^{-}$, and $L^{+}$lines in spectra of different SAQD samples demonstrate pronounced resonances which are shifted by the $E_{L^{-}}$and $E_{L^{+}}$energies with respect to the corresponding 0-0 PL bands (see Fig. 5). This fact indicates that both the PLP modes of doped GaAs substrate are coupled with the QD electronic subsystem and are involved in the intraband carrier relaxation in quantum dot in the same manner as intrinsic phonons of quantum dot and its nearest surroundings. The observed plasmon-phonon lines are therefore superposition of the Raman signals from the substrate and the RPL signals from InAs SAQD's with comparable amplitudes at the maximum of the PLP line excitation profile. Importantly, the intraband carrier relaxation in the quantum dots induced by the plasmon-phonon modes is effective enough although distance between the substrate and QD layer is $100 \mathrm{~nm}$ long.

As is known, ${ }^{15}$ the studied SAQD systems possess an inhomogeneously broadened spectrum of intraband transitions. In particular, light with definite photon energy in the region of the 1-1 transitions excites an ensemble of quantum dots with broad energy spectrum of intraband transitions to the lowest electron-hole states. Phonon-mediated intraband relaxation results in the RPL spectrum with the phonon related peaks. In other words, the RPL spectrum as a function of the Stokes shift can be considered as a spectrum of intraband relaxation rates. In the framework of our model we calculated a dependence of relaxation rate of the lowest energy intraband electronic transitions as a function of the intraband level spacing $\Omega$ (i.e., the spectrum of intraband relaxation rates). The calculations were performed for $z_{0}=100 \mathrm{~nm}$ and different concentrations of the free electrons in region of $n_{0}=10^{18} \mathrm{~cm}^{-3}$. Spherical quantum dot with infinite potential barrier was considered. A relation of $R_{0}$ $=\sqrt{\hbar\left(\xi_{11}^{2}-\pi^{2}\right) /\left(2 m_{Q D} \Omega\right)}$ between the QD radius and $\Omega$, where $m_{Q D}$ is the electronic effective mass in InAs, was used.

Figure 6 shows the results of calculation for $n_{0}=0.8$ $\times 10^{18} \mathrm{~cm}^{-3}$ [solid line in Fig. 6(a)] in comparison with the experimental RPL spectrum of sample A [Fig. 6(b)] in the spectral region of interest. We see that the positions and widths $L^{-}$and $L^{+}$lines in the RPL spectrum are quite close to the calculated energy positions of relaxation windows with widths of several meV. The same correspondence has been observed for the RPL spectra of sample B. A little difference between $n_{0}$ of the substrate and its value used in the calculations may come from the fact that the optical phonon dispersion and/or retardation effects were not taken into account in our model. The relaxation rates for both PLP modes were estimated to be about $2 \times 10^{8} \mathrm{~s}^{-1}$ in spite of the relatively long distance between the quantum dot and doped semicon-

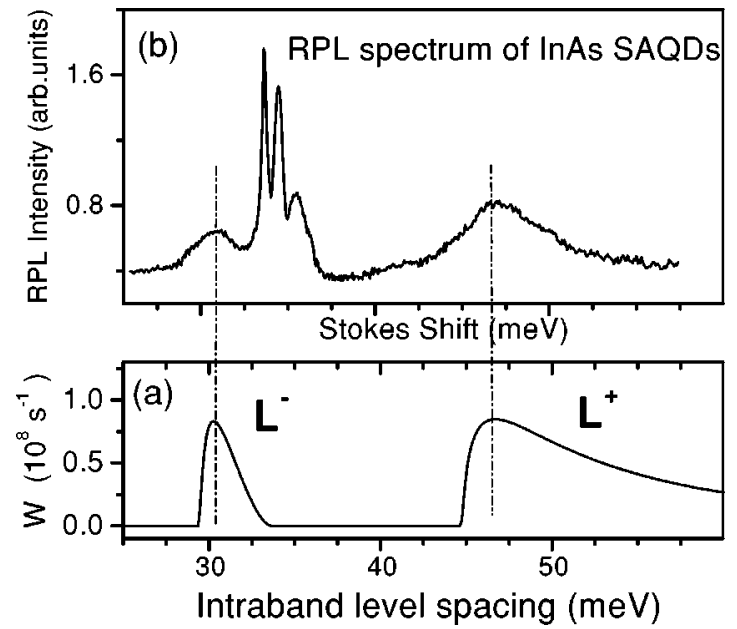

FIG. 6. Comparison between (a) calculated spectrum of relaxation rate $W$ and (b) relevant portion of the RPL spectrum of sample A, the same as shown in Fig. (4) (upper panel). The calculations based on Eq. (17) have been done for the lowest electronic intraband transitions in spherical InAs QD located at distance $z_{0}$ $=100 \mathrm{~nm}$ from $n$-doped GaAs substrate and dopant concentration of $n_{0}=0.8 \times 10^{18} \mathrm{~cm}^{-3} . L^{-}$and $L^{+}$denote the relaxation windows corresponding to the PLP modes of GaAs substrate.

ductor. It is seen that observed features of the RPL spectra are in accordance with predictions of our theoretical model for the intraband carrier relaxation with emission of the plasmon-phonon modes. Indeed, as we have demonstrated by Eq. (17), the coupling between the QD electronic subsystem and plasmon-phonon modes via the self-consisting electric potential opens two relaxation windows corresponding to the QD interaction with the upper and lower PLP branches.

We would like to note that both the peak value and width of the relaxation windows are determined by the PLP
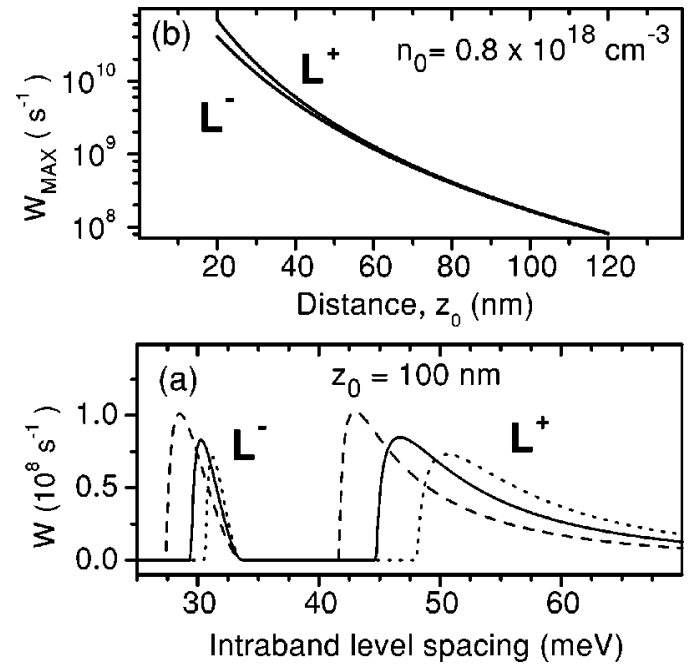

FIG. 7. (a) Calculated relaxation rate for lowest energy intraband electronic transitions $W$ in spherical InAs QD for different dopant concentration in GaAs substrate: $n_{0}=0.6 \times 10^{18} \mathrm{~cm}^{-3}, n_{0}$ $=0.8 \times 10^{18} \mathrm{~cm}^{-3}$, and $n_{0}=10^{18} \mathrm{~cm}^{-3}$ (dashed, solid, and dotted curves, respectively). A distance between the substrate and QD $z_{0}$ $=100 \mathrm{~nm}$. (b) Peak value of the $W\left(W_{M A X}\right)$ as a function of $z_{0}$ for $n_{0}=0.8 \times 10^{18} \mathrm{~cm}^{-3}$. 
mode dispersion, Eq. (12). At the same time, the spectral positions of the windows remarkably depend on the dopant concentration that is shown in Fig. 7(a) by the spectra of the relaxation rates calculated for different dopant concentrations $\left(n_{0}=0.6 \times 10^{18} \mathrm{~cm}^{-3}, n_{0}=0.8 \times 10^{18} \mathrm{~cm}^{-3}\right.$, and $n_{0}$ $\left.=10^{18} \mathrm{~cm}^{-3}\right)$. The spectra demonstrate an $8-\mathrm{meV}$ shift of the $L^{+}$window with $n_{0}$. This fact is very important because the rates can be essentially varied by tuning of the PLP-mode windows to resonance or out of resonance with QD intraband transition energies by means of changing of $n_{0}$. Thus the switch on or switch off of the relaxation channels become possible. Such control of the intraband carrier dynamics will be especially effective in QD systems where inhomogeneous broadening of the intraband transitions is narrower than the window widths, in QD systems without such kind of broadening, and in a single QD. As for the efficiency of the relaxation process, our calculations show that with decrease of distance $z_{0}$ the peak values of the relaxation rates drastically increase [Fig. 7(b)] up to about $10^{11} \mathrm{~s}^{-1}$ at $z_{0}=20 \mathrm{~nm}$. This value is comparable with rate of the Auger-like process of QD interband carrier relaxation. ${ }^{20}$
We believe the considered interaction has to be accounted for in design of QD based applications where $n(p)$-doped structural components of heterostructures spaced apart from the QD layers by ten or several ten nanometers are widely used. The concentration and distance dependencies of the coupling open promising opportunities for manipulation of QD electron dynamics in doped heterostructures. We would like to note that surface plasmon-LO-phonon modes of doped heterostructure components can be also involved in the intraband carrier relaxation in quantum dots. Consideration of this problem is in progress.

\section{ACKNOWLEDGMENTS}

The authors thank Dr. S. Sugou and Dr. H.-W. Ren for growing samples studied in this work. The work was partially supported by the Single Quantum Dot Project, ERATO, JST, Japan which the authors deeply acknowledge. A.V.B. and A.V.F. are grateful to the RFBR (Grant No. 02-0217311) and to the INTAS Program (01-2100 and 01-2331) for partial financial support of this work.
*Electronic address: baranov1@online.ru

${ }^{1}$ L. Guo, E. Leobandung, and S.Y. Chou, Science 275, 649 (1997).

${ }^{2}$ T. Itakura and Y. Tokura, Phys. Rev. B 67, 195320 (2003).

${ }^{3}$ K. Yano, T. Ishii, T. Sano, T. Mine, F. Murai, T. Hashimoto, T. Koboyashi, T. Kure, and K. Seki, Proc. IEEE 87, 633 (1999).

${ }^{4}$ M. Dutta and M. A. Stroscio, Advances in Semiconductor Lasers and Applications to Optoelectronics (World Scientific, Singapore, 2000).

${ }^{5}$ X.-Q. Li and Y. Arakawa, Phys. Rev. B 57, 12285 (1998).

${ }^{6}$ X.-Q. Li, H. Nakayama, and Y. Arakawa, Phys. Rev. B 59, 5069 (1999).

${ }^{7}$ F. Gindele, K. Hild, W. Langbain, and U. Woggon, Phys. Rev. B 60, R2157 (1999).

${ }^{8}$ A.V. Baranov, V. Davydov, H.-W. Ren, S. Sugou, and Y. Masumoto, J. Lumin. 87-89, 503 (2000).

${ }^{9}$ I.V. Ignatiev, I.E. Kozin, S.V. Nair, H.-W. Ren, S. Sugou, and Y. Masumoto, Phys. Rev. B 61, 15633 (2000).

${ }^{10}$ I.V. Ignatiev, I.E. Kozin, V.G. Davydov, S.V. Nair, J.-S. Lee, H.-W. Ren, S. Sugou, and Y. Masumoto, Phys. Rev. B 63, 075316 (2001).

${ }^{11}$ P.A. Knipp and T.L. Reinecke, Phys. Rev. B 46, 10310 (1992).

${ }^{12}$ G. Biese, C. Schüller, K. Keller, C. Steinebach, D. Heitmann, P. Grambow, and K. Eberl, Phys. Rev. B 53, 9565 (1996).

${ }^{13}$ S. Zanier, Y. Guldner, J.P. Vieren, G. Faini, E. Cambril, and Y. Campidelli, Phys. Rev. B 57, 1664 (1998).

${ }^{14}$ T. Ioshita and H. Sakaki, Phys. Rev. B 46, 7260 (1992).

${ }^{15}$ R. Heitz, M. Veit, N.N. Ledentsov, A. Hoffman, D. Bimberg, V.M. Ustinov, P.S. Kop'ev, and Z.I. Alferov, Phys. Rev. B 56, 10435 (1997).

${ }^{16}$ P.C. Sersel, Phys. Rev. B 51, 14532 (1995).

${ }^{17}$ D.F. Schroeter, D.F. Griffits, and P.C. Sersel, Phys. Rev. B 54, 1486 (1996).

${ }^{18}$ X.-Q. Li and Y. Arakawa, Phys. Rev. B 56, 10423 (1997).
${ }^{19}$ U. Bockelman and T. Egeler, Phys. Rev. B 46, 15574 (1992).

${ }^{20}$ A.L. Efros, V.A. Kharchenko, and M. Rosen, Solid State Commun. 93, 281 (1995).

${ }^{21}$ U. Bockelman and G. Bastard, Phys. Rev. B 42, 8947 (1990).

${ }^{22}$ H. Benisty, Phys. Rev. B 51, 13281 (1995).

${ }^{23}$ A.V. Fedorov, A.V. Baranov, and Y. Masumoto, Solid State Commun. 122, 139 (2002).

${ }^{24}$ A.V. Uskov, K. Nishi, and R. Lang, Appl. Phys. Lett. 74, 3081 (1999).

${ }^{25}$ E. Evans and D.L. Mills, Phys. Rev. B 8, 4004 (1973).

${ }^{26}$ N. Mori and T. Ando, Phys. Rev. B 40, 6175 (1989).

${ }^{27}$ B.K. Ridley, Phys. Rev. B 49, 17253 (1994).

${ }^{28}$ F. Comas, C. Trallero-Giner, and M. Cardona, Phys. Rev. B 56, 4115 (1997).

${ }^{29}$ R.H. Ritchie and R.E. Wilems, Phys. Rev. 178, 372 (1969).

${ }^{30}$ L. Kleinman, Phys. Rev. B 7, 2288 (1973).

${ }^{31}$ K.S. Srivastava and A. Tandon, Phys. Rev. B 39, 3885 (1989).

${ }^{32}$ E. Hanamura, Phys. Rev. B 37, 1273 (1988).

${ }^{33}$ D.A. Varshalovich, A. N. Moskalev, and V. K. Hersonskii, Quantum Theory of Angular Moment (World Scientific, Singapore, 1987).

${ }^{34}$ K. Vahala, IEEE J. Quantum Electron. QE-24, 523 (1988).

${ }^{35}$ S. Raymond, X. Guo, J.L. Merz, and S. Fafard, Phys. Rev. B 59, 7624 (1999).

${ }^{36}$ A. Wojs, P. Hawrylak, S. Fafard, and L. Jacak, Phys. Rev. B 54, 5604 (1996).

${ }^{37}$ K.H. Schmidt, G. Medeiros-Ribeiro, M. Oestreich, P.M. Petroff, and G.H. Döhler, Phys. Rev. B 54, 11346 (1996).

${ }^{38}$ A. Mooradian and A.L. McWhorter, Phys. Rev. Lett. 19, 849 (1967).

${ }^{39}$ G. Abstreiter, M. Cardona, and A. Pinczuk, in Light Scattering in Solids IV, edited by M. Cardona and G. Guntherodt (Springer, Berlin, 1984). 